\title{
EFEITO DO ENVELHECIMENTO DE AGUARDENTES NAS CARACTERÍSTICAS SENSORIAIS E PREFERÊNCIA
}

\author{
M.R. Verruma-Bernardi ${ }^{1}$, C. Parazzi ${ }^{1}$, M.T.M.R. Borges ${ }^{1}$, V.M. Macedo ${ }^{2}$, \\ K.S. Ferreira ${ }^{2}$, R. Deliza ${ }^{3}$
}

\begin{abstract}
RESUMO
O trabalho teve como objetivo descrever sensorialmente aguardentes armazenadas em tonéis de carvalho durante seis períodos, a saber: no tempo zero, aos 12, 24, 48,72 e 96 meses, bem como a preferência do consumidor nos respectivos períodos e os parâmetros físico-químicos. As aguardentes utilizadas no experimento foram coletadas em unidade produtora na região de Araras - SP, proveniente de processo de destilação em colunas de fluxo contínuo. Oito provadores selecionados e treinados participaram da Análise Descritiva Quantitativa e a avaliação da preferência foi realizada com 148 indivíduos. A ordem de apresentação das seis amostras foi balanceada e seguiu delineamento de blocos completos. Os dados foram analisados por análise de variância e posterior teste Tukey para checar diferença entre as médias $(p \leq 0,05)$ assim como por análise de componentes principais utilizando o programa estatístico SAS. Os atributos sensoriais que descreveram as cachaças foram: aparência (coloração amarelada), aroma (madeira, adocicado, alcoólico), sabor (adocicado, ácido, agressividade, adstringência, alcoólico). De acordo com os resultados obtidos verificou-se que não ocorreram diferenças estatísticas $(\mathrm{p} \geq 0,05)$ entre as aguardentes para os atributos: aroma alcoólico, gosto adocicado, gosto ácido e agressividade. Entretanto a coloração amarelada, aroma de madeira e aroma adocicado intensificaram-se com o armazenamento $(\mathrm{p} \leq 0,05)$ enquanto a adstringência e o sabor alcoólico diminuíram para as amostras com maior tempo de armazenamento. Os consumidores apreciaram as aguardentes mais envelhecidas em relação à aparência, aroma, sabor e aceitação global.
\end{abstract}

Palavras-chave: armazenamento, preferência, análise descritiva quantitativa, cachaça.

\section{QUANTITATIVE DESCRIPTIVE AND PREFERENCE ANALYSIS FOR AGED SPIRITS}

\section{ABSTRACT}

The study aimed at describing the sensory characteristics and investigating the consumer preference for cachaça aged in oak barrels during six periods, as follows: zero, 12, 24, 48, 72 and 96 months. The cachaças used in this study came from a production unit located in the region of Araras - SP, and they were obtained from a continuous flow distillation process. The Quantitative Descriptive Analysis method was carried out with eight selected and trained panelists and the preference test was conducted with 148 cachaça's consumers. The samples' presentation order was balanced and followed a complete block design. The data were analyzed through the ANOVA followed by the Tukey test to check difference among means $(p<0.05)$ and the principal component analysis using the software SAS. The sensory attributes that described the cachaça were: appearance (yellowing color), aroma (wood, sweet, alcoholic), flavor (sweet, sour, astringency aggressive, alcoholic). There were no statistical differences $(\mathrm{p} \geq 0.05)$ among the cachaças for the attributes alcoholic aroma, sweet taste, taste sour, and aggressiveness. However the yellowing color, wood aroma and sweet aroma increased with the storage $(\mathrm{p}<0.05)$. On the other hand, the astringency and alcoholic flavor decreased. Consumers liked the aged cachaças in relation to the appearance, aroma, flavor and overall acceptance.

Keywords: storage, preference, quantitative descriptive analysis, cachaça.

\footnotetext{
Protocolo

${ }^{1}$ Departamento de Tecnologia Agroindustrial e Sócio-Economia Rural/UFSCar. Via Anhanguera, km 174 - Caixa Postal 153, 13600-970 - Araras/SP. E-mail: verruma@cca.ufscar.br

${ }^{2}$ Alunas do Curso de Engenharia Agronômica - UFSCar

${ }^{3}$ Embrapa Agroindústria de Alimentos. Av. das Américas, 29501, 23020.470 Rio de Janeiro - RJ.
} 


\section{INTRODUÇÃO}

A denominação aguardente de cana refere-se à bebida com graduação alcoólica de 38 a $54 \%$, em volume, à $20^{\circ} \mathrm{C}$, obtida pela destilação simples do mosto fermentado do caldo de cana-de-açúcar (Saccharum officinarum), podendo ser adicionada açucares até $6 \mathrm{~g} / \mathrm{L}$, expressos em sacarose (Brasil, 2005).

A aguardente de cana é muito apreciada por seu aroma e sabor característicos que podem ainda ser melhorados pelo envelhecimento em recipientes de madeira. O complexo processo que ocorre durante o envelhecimento depende de vários fatores, entre eles do tipo de madeira empregada, do tempo de maturação e obviamente da qualidade inicial do destilado.

Para Cardello \& Faria (1999) o envelhecimento da aguardente em tonéis de madeira proporciona uma efetiva melhora sensorial no produto, pois promove diminuição significativa do sabor alcoólico e da agressividade da bebida, com aumento simultâneo da doçura e do sabor da madeira. Cardello \& Faria (1998) relataram que todas as etapas do preparo de bebidas destiladas são determinantes e podem influenciar o desenvolvimento de aromas.

O objetivo deste trabalho foi avaliar parâmetros físico-químicos e sensoriais de aguardentes de uma unidade produtora na região de Araras, SP, proveniente de processo de destilação em colunas de fluxo contínuo, armazenadas por oito anos.

\section{MATERIAL E MÉTODOS}

\section{Material}

Foram analisadas seis aguardentes de uma unidade produtora da região de Araras SP, proveniente de processo de destilação em colunas de fluxo contínuo. As aguardentes foram processadas gradativamente e armazenadas em tonéis de carvalho por zero, 12, 24, 48, 72 e 96 meses.

\section{Análises físico-químicas}

Os parâmetros analisados foram acidez, teor alcoólico $\left({ }^{\circ} \mathrm{GL}\right)$ e cinzas condutimétricas (Amorim et al., 1979). As determinações de cobre foram realizadas por espectrofotometria de absorção atômica em chama (Rodella \& Borges, 1989) e foram realizadas no Laboratório de Análises e Simulação Tecnológica (LAST) do Departamento de Tecnologia Agroindustrial e Sócio-Economia Rural do Centro de Ciências Agrárias da UFSCar. As análises foram realizadas em triplicata.

\section{Análise Descritiva Quantitativa (ADQ)}

A Análise Descritiva Quantitativa foi adaptada segundo Stone \& Sidel (1985), Os testes foram realizados no horário de 14:00 às 17:00 horas.

Participaram deste estudo 14 provadores pré-selecionados (8) mulheres e 7 homens com idade entre 25 e 50 anos) levandose em consideração $o$ interesse e disponibilidade no período de realização da análise, além do conhecimento prévio em análise sensorial. Cerca de $20 \mathrm{~mL}$ das amostras de aguardentes (zero x 96 meses, 12 × 72 meses, $24 \times 48$ meses) foram servidas à temperatura ambiente em taças codificadas com números de três dígitos para o levantamento de atributos, o qual utilizou o método Rede"Kelly's Repertory Grid Method" (Moskowitz, 1983). Foram realizadas três sessões onde foi apresentado um par de amostras de aguardente por vez, solicitando-se que o provador anotasse os atributos, as similaridades e as diferenças entre elas, utilizando ficha adequada.

Após cada provador realizar o levantamento de termos descritivos para os pares de amostras, a equipe se reuniu e discutiu sobre os termos elucidados. Nesta etapa, aqueles que expressaram o mesmo significado foram agrupados em um só atributo. Já os poucos utilizados pelos provadores foram retirados. No final das sessões foi gerada, por consenso, uma lista de termos descritivos com os respectivos extremos da cada escala utilizada bem como as respectivas definições. Durante o treinamento os provadores foram solicitados a avaliar a intensidade de cada atributo sensorial das amostras de aguardentes. Para a avaliação foram utilizadas escalas não estruturadas de $10 \mathrm{~cm}$, variando de zero (ausência) a 9 (forte) ancorada nos extremos com termos definidos pela equipe. A lista dos atributos com as respectivas definições e extremos utilizados é mostrada na Tabela 1. 
Tabela 1. Termos descritivos utilizados, respectivas definições e referências

\begin{tabular}{|c|c|c|}
\hline Atributos & Definições & Referências \\
\hline Aparência & $\begin{array}{l}\text { Coloração amarelada: refere-se à cor de } \\
\text { cerveja. }\end{array}$ & $\begin{array}{l}\text { Ausente: água destilada. } \\
\text { Forte: Whisky. }\end{array}$ \\
\hline \multirow[t]{3}{*}{ Aroma } & $\begin{array}{l}\text { Madeira: refere-se ao aroma característico } \\
\text { da madeira do carvalho utilizado no tonél } \\
\text { para envelhecimento das aguardentes. }\end{array}$ & $\begin{array}{l}\text { Ausente: etanol. } \\
\text { Forte: presença do aroma de } \\
\text { madeira de carvalho. }\end{array}$ \\
\hline & $\begin{array}{l}\text { Adocicado: doce característico de } \\
\text { aguardente permanecendo por certo tempo } \\
\text { após a ingestão }\end{array}$ & $\begin{array}{l}\text { Ausente: etanol. } \\
\text { Forte: aroma de etanol com } \\
\text { presença do aroma doce. }\end{array}$ \\
\hline & Alcoólico: Aroma característico do etanol. & $\begin{array}{l}\text { Fraco: etanol a } 36 \% \text {. } \\
\text { Forte: etanol a } 42 \%\end{array}$ \\
\hline
\end{tabular}

Sabor

Adocicado: gosto doce que permanece por um período de tempo.

Ácido: gosto ácido característico de frutas cítricas.

Agressividade: impacto agressivo inicial.

Alcoólico: sabor de álcool percebido no momento que ingere a bebida, permanecendo por certo período na boca.
Ausente: etanol.

Forte: gosto de etanol com presença do sabor doce.

Fraco: sabor suave sem sensação do sabor agressivo.

Forte: sabor de etanol a $45 \%$.

Ausente: água destilada

Muito: cachaça com $45 \%$ de etanol.

Fraco: cachaça $37 \%$ de etanol. Forte: cachaça $45 \%$ de etanol.

Adstringência: sensação produzida na mordida de frutas verdes, que dá a sensação § que "amarra" a boca.

\footnotetext{
* os provadores não apresentaram referencias para estes atributos.
}

Após o treinamento os provadores avaliaram as amostras apresentadas codificadas com número de três dígitos com três repetições utilizando a ficha desenvolvida. Foram selecionados aqueles que demonstraram habilidade em relação a discriminar as amostras. A ordem de apresentação das amostras foi balanceada. Água e biscoito de água e sal foram fornecidos à equipe para a limpeza do palato entre as amostras.

\section{Avaliação da preferência}

Foi realizada a avaliação da preferência em relação à aparência, aroma, sabor e aceitação geral, utilizando-se escala hedônica de sete pontos variando de "desgostei muito" a "gostei muito". Cento e quarenta e oito indivíduos (148 homens com idade variando de 25 e 50 anos) que gostam e consomem cachaça participaram do teste. As amostras foram apresentadas codificadas com números de três dígitos em copos para cachaça à temperatura ambiente e a ordem de apresentação foi balanceada seguindo o delineamento de blocos completos sugerido por MacFIE et al. (1989).

\section{Análise estatística}

Os dados da análise sensorial foram analisados por ANOVA seguido do teste de Tukey para checar diferença entre as médias e analise dos componentes principais utilizando o programa SAS (2003). 


\section{RESULTADOS E DISCUSSÃO}

\section{Análises físico-químicas}

A Tabela 2 apresenta os resultados de: acidez, teor alcoólico e cinzas obtidos para as aguardentes analisadas. Os resultados de teor alcoólico variaram de $38,00-39,38^{\circ} \mathrm{GL}$. A legislação brasileira estabelece os limites de 38,0 a 54,0GL, sendo assim as amostras encontraram-se de acordo com as especificações (Brasil, 2005).

Para os teores de acidez, observou-se uma variação entre 4,5 e $8,9 \mathrm{mg} / 100 \mathrm{~mL}$. De acordo com Cardello \& Faria (1997) e Souza \& Del Mastro (2000) durante a maturação de bebidas destiladas normalmente ocorre uma diminuição do pH e das concentrações de álcool metílico e de álcool etílico, enquanto há um aumento da acidez, da cor e das concentrações de acetato de etila, de aldeído acético, de acetona e dos compostos fenólicos. Estudos descritos por Parazzi et al. (2008) mostraram que os recipientes utilizados e o tempo de armazenamento interferiram nas características químicas e na qualidade da aguardente de canade-açúcar.

A avaliação de cinzas indicou a presença de sais solúveis. Observou-se um aumento ao longo do armazenamento. As concentrações de cobre nas amostras foram inferiores ao limite máximo exigido pela legislação, ou seja, $5 \mathrm{ppm}$ (Brasil, 2005), variando de 1,07 a 1,57.

Tabela 2. Valores médios ${ }^{\S}$ dos parâmetros físico-químicos das aguardentes.

$\begin{array}{ccccc}\begin{array}{c}\text { Aguardente } \\ \begin{array}{c}\text { Armazenamento } \\ \text { (meses) }\end{array}\end{array} & \begin{array}{c}\text { Teor alcoólico } \\ \left({ }^{\circ} \mathrm{GL}\right)\end{array} & \begin{array}{c}\text { Acidez } \\ (\mathrm{mg} / 100 \mathrm{~mL})\end{array} & \begin{array}{c}\text { Cinzas } \\ (\propto \mathrm{S})\end{array} & \begin{array}{c}\text { Cobre } \\ (\mathrm{ppm})\end{array} \\ 0 & 39,38 & 4,5 & 45,5 \\ 12 & 38,31 & 7,4 & 38,6 \\ 24 & 38,00 & 5,1 & 32,1 \\ 48 & 38,25 & 8,9 & 49,8 \\ 72 & 38,00 & 8,9 & 49,3 \\ 96 & 38,89 & 8,9 & 52,2\end{array}$

\section{Análise sensorial}

Dentre os 14 indivíduos préselecionados, oito foram considerados aptos e os resultados apresentados a seguir. Os atributos sensoriais que descreveram as aguardentes foram: aparência (coloração amarelada), aroma (madeira, adocicado e alcoólico) e sabor (gosto adocicado, gosto ácido, agressividade, alcoólico e adstringência).

A análise de variância dos dados sensoriais para cada atributo indicou diferença significativa $(\mathrm{p} \leq 0,05)$ para a maioria dos termos descritivos avaliados pelos oito provadores selecionados (Tabela 3).

Tabela 3. Médias dos atributos sensoriais ${ }^{\S}$ das seis amostras de aguardentes avaliados.

\begin{tabular}{lccccccr}
\hline \multicolumn{7}{c}{ Atributos sensoriais } & \multicolumn{7}{c}{ Meses de armazenamento } & \multicolumn{1}{c}{ DMS* } \\
\hline Coloração & 0 & 12 & 24 & 48 & 72 & 96 & \\
Aroma madeira & $2,40 \mathrm{c}$ & $1,77 \mathrm{c}$ & $1,90 \mathrm{c}$ & $6,43 \mathrm{~b}$ & $7,26 \mathrm{a}$ & $6,81 \mathrm{ab}$ & 0,805 \\
Aroma adocicado & $3,09 \mathrm{bc}$ & $2,31 \mathrm{bc}$ & $2,19 \mathrm{c}$ & $3,64 \mathrm{~b}$ & $5,92 \mathrm{a}$ & $6,27 \mathrm{a}$ & 1,3508 \\
Aroma alcoólico & $3,12 \mathrm{bc}$ & $2,85 \mathrm{c}$ & $3,58 \mathrm{abc}$ & $4,89 \mathrm{a}$ & $4,29 \mathrm{ab}$ & $4,37 \mathrm{ab}$ & 1,3635 \\
Gosto adocicado & $4,77 \mathrm{a}$ & $4,48 \mathrm{a}$ & $3,82 \mathrm{a}$ & $4,28 \mathrm{a}$ & $4,75 \mathrm{a}$ & $4,69 \mathrm{a}$ & 1,6454 \\
Gosto ácido & $3,11 \mathrm{a}$ & $3,32 \mathrm{a}$ & $3,59 \mathrm{a}$ & $3,87 \mathrm{a}$ & $3,12 \mathrm{a}$ & $4,02 \mathrm{a}$ & 1,5618 \\
Sabor agressivo & $4,53 \mathrm{ab}$ & $3,38 \mathrm{~b}$ & $3,89 \mathrm{ab}$ & $4,20 \mathrm{ab}$ & $4,85 \mathrm{a}$ & $3,95 \mathrm{ab}$ & 1,2597 \\
Sabor alcoólico & $5,93 \mathrm{a}$ & $5,15 \mathrm{a}$ & $5,75 \mathrm{a}$ & $5,08 \mathrm{a}$ & $4,55 \mathrm{a}$ & $4,77 \mathrm{a}$ & 1,4857 \\
Adstringência & $5,75 \mathrm{ab}$ & $5,55 \mathrm{ab}$ & $5,70 \mathrm{ab}$ & $5,18 \mathrm{~b}$ & $6,58 \mathrm{a}$ & $5,19 \mathrm{~b}$ & 1,2419 \\
\hline & $5,42 \mathrm{a}$ & $4,27 \mathrm{ab}$ & $4,70 \mathrm{ab}$ & $5,00 \mathrm{ab}$ & $5,57 \mathrm{a}$ & $3,72 \mathrm{~b}$ & 1,5935 \\
\hline
\end{tabular}

Médias com letras diferentes indicam diferenças significativas $(\mathrm{p} \leq 0,05) .{ }^{\S}$ avaliados em escala não estruturada variando de $0=$ ausente a $9=$ forte.

*Diferença mínima significativa ao nível de 5\%. 
A cor das aguardentes foi avaliada no intervalo "ausente a forte" e os provadores utilizaram praticamente toda escala para avaliar as amostras. A partir dos48 meses de armazenamento a coloração ficou mais intensa.

As aguardente com maior tempo de armazenamento (72 e 96 meses) apresentaramse mais intensas em relação ao aroma de madeira com valores 5,92 e 6,27, respectivamente. Para o aroma adocicado os valores foram aumentando à medida que houve o aumento de tempo de armazenamento, porém o uso da escala foi restrito entre 2,85 (amostra armazenada 12 meses) e 4,37 (amostra com 96 meses). Não houve diferença significativa $(\mathrm{p} \geq 0,05)$ entre as aguardentes em relaçao ao aroma alcoólico.
Para os atributos de sabor verificou-se que houve os provadores utilizaram a mesma parte da escala. Para adstringência verificou-se diferença significativa entre as amostras no tempo zero e aos 96 meses armazenadas.

A Análise de Componentes Principais (ACP) é ferramenta extremamente útil na análise de dados sensoriais (Borgognone et al., 2001), pois permite visualizar e analisar correlações entre as diversas variáveis do estudo. A Figura 1 mostra a posição das amostras de aguardentes e a distribuição dos atributos sensoriais no espaço definido pela primeira e segunda dimensões. Verificou-se que os Componentes Principais F1 e F2 explicam $80,62 \%$ das variações entre as amostras.

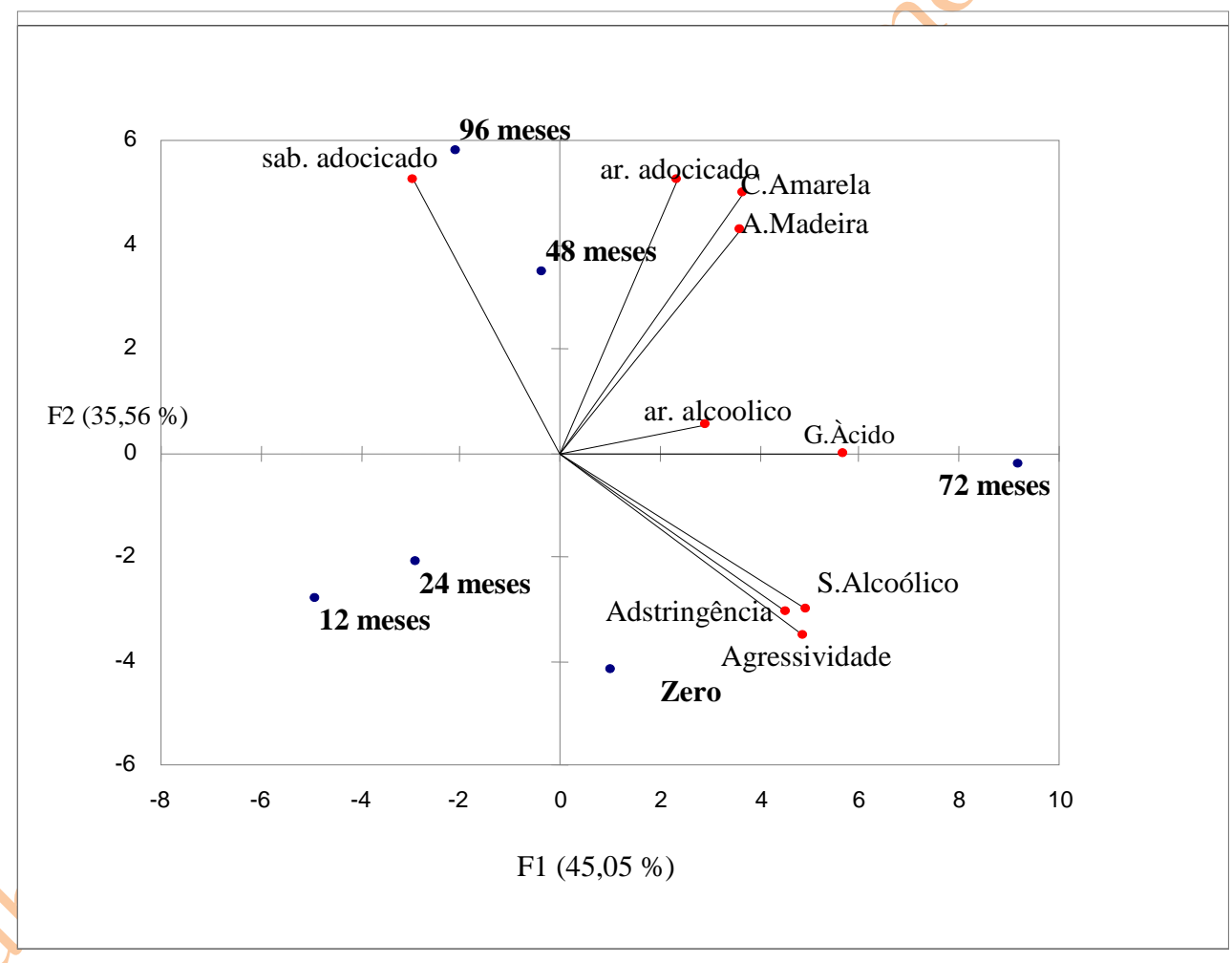

Figura 1. Análise de componentes principais (ACP) das aguardentes - posição dos atributos sensoriais e amostras.

\section{Avaliação da preferência das aguardentes}

De acordo com os resultados obtidos verificou-se que as aguardentes mais envelhecidas foram as preferidas em relação ao quanto os consumidores gostaram da aparência, aroma, sabor e a aceitação geral (Tabela 4). 
Tabela 4. Médias ${ }^{\S}$ de preferência das aguardentes estudadas.

\begin{tabular}{ccccc}
\hline $\begin{array}{c}\text { Aguardentes } \\
\begin{array}{c}\text { Armazenamento } \\
\text { (meses) }\end{array}\end{array}$ & Aparência & Aroma & Sabor & Aceitação Global \\
0 & $4,7 \mathrm{c}$ & $4,3 \mathrm{~b}$ & $3,8 \mathrm{~b}$ & $4,7 \mathrm{~b}$ \\
12 & $5,4 \mathrm{~b}$ & $4,8 \mathrm{ab}$ & $4,6 \mathrm{a}$ & $4,9 \mathrm{a}$ \\
24 & $5,4 \mathrm{~b}$ & $4,7 \mathrm{ab}$ & $4,7 \mathrm{a}$ & $4,9 \mathrm{a}$ \\
48 & $5,7 \mathrm{ab}$ & $5,2 \mathrm{a}$ & $4,8 \mathrm{a}$ & $5,2 \mathrm{a}$ \\
72 & $5,9 \mathrm{a}$ & $5,2 \mathrm{a}$ & $4,8 \mathrm{a}$ & $5,2 \mathrm{a}$ \\
96 & $6,0 \mathrm{a}$ & $5,2 \mathrm{a}$ & $5,0 \mathrm{a}$ & $5,3 \mathrm{a}$ \\
\hline *DMS & 0,437 & 0,5517 & 0,6281 & 0,5267 \\
\hline
\end{tabular}

Médias com letras diferentes indicam diferença significativa $(\mathrm{p} \leq 0,05) .{ }^{{ }^{\S}}$ avaliadas em escala hedônica de sete pontos, variando de $1=$ desgostei muito a $7=$ gostei muito.

\section{CONCLUSÕES}

O envelhecimento a partir dos 48 meses favoreceu o desenvolvimento de alguns atributos sensoriais os quais devem ter contribuído para aceitação das aguardentes.

\section{REFERÊNCIAS BIBLIOGRÁFICAS}

Amorim, H.V.; Zago, E.A.; Gutierrez, L.E. Método rápido para controle da fermentação e destilação. Saccharum/STAB, v.2, n.4, p.31-34. 1979.

Borgognone, M. G.; Bussi, J.; Hough, G. Principal component analysis in sensory analysis: covariance or correlation matrix? Food Quality and Preference, v.12, p.323326, 2001.

Brasil. Leis, decretos, etc. Instrução Normativa $\mathrm{n}^{\circ} 13$ de 29 de junho de 2005. Diário Oficial da União - Ministério da Agricultura, Pecuária e Abastecimento. Brasília - DF, 30 jun.2005. Seção 1, p. 3-4.

Cardello, H.M.A.B.; Faria, J.B. Análise tempointensidade de características sensoriais de cachaça de cana durante o envelhecimento em tonel de carvalho (Quercus sp.). Boletim da Sociedade Brasileira de Ciência e Tecnologia de Alimentos, v.33, n.1, p.2734. 1999.

Cardello, H.M.A.B.; Faria, J.B. Modificações físico-químicas e sensoriais de cachaça de cana durante o envelhecimento em tonel de carvalho (Quercus alba L.). Boletim do Centro de Pesquisa e Processamento de Alimentos, Curitiba, v.15, n.2, p. 87-100, 1997.
Cardello, H.M.A.B.; Faria, J.B. Análise descritiva quantitativa da aguardente de cana durante o envelhecimento em tonel de carvalho (Quercus alba 1.). Ciência e Tecnologia de Alimentos, Campinas, v.8, n. 2, 1998

Behrens. J.H.; Silva, M.A.A.P. Da; Wakeling, I.N. Avaliação da aceitação de vinhos brancos varietais brasileiros através de testes sensoriais afetivos e técnica multivariada de mapa de preferência interno. Ciência e Tecnologia de Alimentos, Campinas, v.19, n.2, p.214-220, 1999.

MacFie, H.J.; N.; Bratchell, N.; Greenhoff, K.; Vallis, L. Designs to balance the effect of order of presentation and first-order carryover effects in hall tests. Journal of Sensory Studies, v.4, n.2, p.129-148, 1989

Moskowitz, H.R. Product testing and sensory evaluation of foods. Westport: Food \& Nutrition, 1983. 605p.

Parazzi, C.; Arthur, C.M.; Lopes, J.J.C.; Borges, M.T.M.R. Avaliação e caracterização dos principais compostos químicos da aguardente de cana-de-açúcar envelhecida em tonéis de carvalho (Quercus sp.). Ciência e Tecnologia de Alimentos, Campinas, v.28, n.1, p. 193-199, 2008.

Souza, M.D.C.A.; Del Mastro, N.L. Irradiation of cane sugar spirit. Radiation Physics and Chemistry, v.57, p. 257-259, 2000.

Rodella, A.A.; Borges, M.T.M.R. Manual básico para o laboratório sucroalcooleiro, Piracicaba, 225p. 1989.

Stone, H.S.; Sidel, J.L. Sensory evaluation practices. London: Academic, 1985. 311p. 